\title{
The Evaluation and Estimation of the Coefficients in the Chebyshev Series Expansion of a Function]
}

\author{
By David Elliott
}

1. Introduction. Suppose $f(x)$ is a function defined for $-1 \leqq x \leqq 1$, and $T_{n}(x)$ is the Chebyshev polynomial of the first kind of degree $n$, defined by

$$
T_{n}(x)=\cos n \theta \text { with } x=\cos \theta .
$$

If $f(x)$ is of bounded variation in $[-1,1]$ then we have

$$
f(x)=\sum_{n=0}^{\infty} a_{n} T_{n}(x)
$$

where $\sum^{\prime}$ denotes a sum whose first term is halved. The coefficients $a_{n}$ are given by

$$
a_{n}=\frac{2}{\pi} \int_{-1}^{1} \frac{f(x) T_{n}(x)}{\sqrt{1-x^{2}}} d x \quad \text { for } \quad n=0,1,2, \cdots .
$$

Recent investigations have considered the application of Chebyshev series to finding numerical solutions to frequently occurring problems. The quadrature problem has been considered by Clenshaw and Curtis [1]; the numerical solution of ordinary differential equations by Clenshaw [2], Fox [3] and Clenshaw and Norton [4]; the numerical solution of Fredholm integral equations by the author [5] and [6]; and finally a simple partial differential equation by the author [7]. In many of these applications it is useful to be able to estimate the degree $N$ of the polynomial approximation to a given function $f(x)$, so that $f(x)$ is then represented to within some prescribed accuracy. In order to do this some knowledge of the magnitude of the coefficients $a_{n}$ is required, in general for large values of $n$. An attempt has been made to do this by the author [6], but the estimates given there are in general fairly conservative. As an example, it is shown that if $f(x)$ is infinitely differentiable in $-1 \leqq x \leqq 1$ with $\left|f^{(n)}(x)\right| \leqq Q_{n}$ then

$$
\left|a_{n}\right| \leqq \frac{Q_{n}}{2^{n-1} n !}
$$

Although this gives a good estimate for functions like $\sin \frac{1}{2} \pi x$ and $e^{x}$, the estimate is poor for a function like $f(x)=4 /(5+4 x)$. Then $Q_{n}=2^{2 n+2} n$ ! and equation (4) gives $\left|a_{n}\right| \leqq 2^{n+3}$ which although true is useless for computational purposes since in fact,

$$
a_{n}=\frac{8 \cdot(-1)^{n}}{3 \cdot 2^{n}}
$$$$
\text { for } n \geqq 0 \text {. }
$$

Equation (3) appears to be unsuited for estimating $a_{n}$ for large values of $n$. It is the purpose of this paper to consider an alternative definition for $a_{n}$ in terms of a contour integral. This definition can then be used to estimate $a_{n}$ in cases when

Received February 26, 1963, revised September 12, 1963.

This research was supported by the U. S. Air Force Office of Scientific Research. 
$f(z)(z=x+i y)$ has poles in the complex plane, is an entire function, is regular except at the points $z= \pm 1$, and has a branch point in the complex plane. These estimates are shown to be very good in many cases.

2. An Alternative Definition of $a_{n}$. Consider the function $f(z)$ where $z=x+i y$. By Cauchy's integral formula, we can represent $f(x)$ by

$$
f(x)=\frac{1}{2 \pi i} \int_{C} \frac{f(z) d z}{z-x}
$$

where $C$ is any contour on and within which $f(z)$ is regular. If, in the first instance, the contour $C$ is chosen so that it contains the interval $-1 \leqq x \leqq 1$, then equation (5) can be substituted into equation (3) to give

$$
a_{n}=\frac{1}{\pi^{2} i} \int_{C} f(z)\left\{\int_{-1}^{1} \frac{T_{n}(x) d x}{\sqrt{1-x^{2}}(z-x)}\right\} d z
$$

the inversion of the integrals being permitted under these conditions. Writing $x=\cos \theta$, it can easily be shown that

$$
\int_{-1}^{1} \frac{T_{n}(x) d x}{\sqrt{1-x^{2}}(z-x)}=\frac{\pi}{\sqrt{z^{2}-1}\left(z \pm \sqrt{z^{2}-1}\right)^{n}}
$$

where the sign is chosen so that $\left|z \pm \sqrt{z^{2}-1}\right|>1$. Then,

$$
a_{n}=\frac{1}{\pi i} \int_{C} \frac{f(z) d z}{\sqrt{z^{2}-1}\left(z \pm \sqrt{z^{2}-1}\right)^{n}} .
$$

Equation (8) is the starting point for all the results of this paper. The choice of the contour $C$, and the evaluation of the contour integral will depend upon the behavior of $f(z)$.

As a first example, let us consider the case where $f(z)$ has a simple pole at the point $z=z_{1}$. We first note that the equation $\left|z \pm \sqrt{z^{2}-1}\right|=\rho$, a constant $>1$, represents an ellipse $E_{\rho}$ with foci at the points $z= \pm 1$, and with semi-axes $a(\rho), b(\rho)$ given by

$$
a(\rho)=\frac{1}{2}\left(\rho+\rho^{-1}\right) \text { and } b(\rho)=\frac{1}{2}\left(\rho-\rho^{-1}\right) .
$$

As the value of $\rho$ varies, we obtain a system of confocal ellipses, the segment $-1 \leqq x \leqq 1$ corresponding to the degenerate case as $\rho \rightarrow 1$. As $\rho$ increases, the semiaxes of the ellipse increase beyond all bounds. Returning to the problem of a function $f(z)$ with a pole at $z=z_{1}$, suppose that this point lies on the ellipse $E_{\rho_{1}}$. Then for our contour $C$, we could choose any ellipse $E_{\rho}$ where $1<\rho<\rho_{1}$. However to evaluate the integral we choose as the contour $C$, an ellipse $E_{\rho}$ where $\rho>\rho_{1}$, described in the positive (counter-clockwise) direction together with a small circle $\gamma_{1}$ with centre at $z_{1}$, described in the negative direction and a pair of lines joining two adjacent points on $\gamma_{1}$, to two adjacent points on $E_{\rho}$ and not intersecting the interval $-1 \leqq x \leqq 1$. Suppose $f(z)$ has a residue $r_{1}$ at the point $z=z_{1}$. To evaluate the contour integral, we let $\rho \rightarrow \infty$ and the radius of the circle $\gamma_{1}$, tend to zero. If $f(z)$ is such that the integral around $E_{\rho}$ tends to zero as $\rho \rightarrow \infty$ for all $n$, we have immediately that 


$$
a_{n}=-\frac{2 r_{1}}{\sqrt{z_{1}^{2}-1}\left(z_{1} \pm \sqrt{z_{1}^{2}-1}\right)^{n}},
$$

valid for $n \geqq 0$. It may happen however, that the integral around $E_{\rho}$ only converges for values of $n$ greater than some $n_{0}$. In this case equation (9) will only be valid for $n>n_{0}$. For a given $n$, the integral over $E_{\rho}$ will tend to zero as $\rho \rightarrow \infty$ provided $\max _{E_{\rho}}|f(z)|=o\left(\rho^{n}\right)$, where $\max _{E_{\rho}}|f(z)|$ denotes the maximum value of $|f(z)|$ on the contour $E_{\rho}$. This will certainly be true if $|f(z)|=o\left(|z|^{n}\right)$ as $|z| \rightarrow \infty$.

This analysis can be generalized immediately to the case where $f(z)$ has a finite number $(M)$ of poles at the points $z_{m}$ for $m=1(1) M$. If we now choose the ellipse $E_{\rho}$ to enclose all these poles, and if as before the integral over $E_{\rho}$ tends to zero as $\rho \rightarrow \infty$, we have,

$$
a_{n}=-2 \sum_{m=1}^{M} \frac{r_{m}}{\sqrt{z_{m}^{2}-1}\left(z_{m} \pm \sqrt{z_{m}^{2}-1}\right)^{n}},
$$

where $r_{m}$ is the residue of $f(z)$ at the point $z_{m}$. Finally, if $f(z)$ is a meromorphic function with an infinity of simple poles, then if the ellipse $E_{\rho}$ is chosen so that no pole of $f(z)$ lies on it, and the integral over $E_{\rho}$ tends to zero as $\rho \rightarrow \infty$ through a sequence of discrete values, we can let $M$ tend to infinity in equation (10). The formulation is useful in this case for estimating the coefficients $a_{n}$ for large $n$, since the contribution from each pole can then be found. An example of this estimation for such a function is given in Section 3.

Returning to the function $f(z)=4 /(5+4 z)$, this has a simple pole at $z_{1}=$ $-5 / 4$, with a residue of 1 . Equation (9) gives immediately that $a_{n}=\frac{8}{3} \cdot \frac{(-1)^{n}}{2^{n}}$, which is exact.

It should be noticed that this technique for evaluating the contour integral in equation (8) is not valid for entire (integral) functions. In Section 5, we consider a technique by which estimates may be obtained for the upper bound of $\left|a_{n}\right|$ for such functions.

3. An Example of a Function With an Infinity of Poles. In [6], the author considered the function

$$
f(z)=\frac{k}{\left(k^{2}+1\right)-\left(k^{2}-1\right) \cos \pi(\alpha+z)}
$$

where $k$ and $\alpha$ are constants such that $k>1$ and $-1 \leqq \alpha \leqq 1$. The problem to be considered consists of finding, for a given value of $k$, that value of $\alpha$ for which the Chebyshev expansion of $f(x)$ has the slowest convergence of the coefficients $a_{n}$, and estimating $a_{n}$ in that case.

The function $f(z)$ has simple poles at the points

$$
z_{m}=(2 m-\alpha) \pm i \beta \quad \text { for } \quad m=0, \pm 1, \pm 2, \cdots
$$

where $\beta=\frac{1}{\pi} \operatorname{arcosh} \frac{k^{2}+1}{k^{2}-1}$. Thus the poles lie along two lines parallel to the real axis and at a distance $\beta$ from it. For a given $m$, each pair of poles corresponding to $\pm i \beta$, lies on an ellipse $E_{\rho_{m}}$ where $\rho_{m}=\left|z_{m} \pm \sqrt{z_{m}{ }^{2}-1}\right|$. The residue of $f(z)$ at 
TABLE 1

\begin{tabular}{l|l|l}
\hline$r$ & estimated $a_{2 r}$ & actual $a_{2 r}$ \\
\hline 2 & +0.03031 & +0.03237 \\
3 & -0.00743 & +0.00739 \\
4 & +0.00183 & +0.00181 \\
5 & -0.000445 & +0.000445 \\
6 & +0.0001088 & +0.001088 \\
\hline
\end{tabular}

$z_{m}$ is $-i / 2 \pi$ when $\operatorname{Im} z_{m}>0$, and $+i / 2 \pi$ when $\operatorname{Im} z_{m}<0$, and is therefore independent of $m$. Thus the largest contribution to $a_{n}$ comes from the poles at $z=$ $-\alpha \pm i \beta$. This, in turn, will be a maximum when $\rho_{0}$ is a minimum i.e. when $\alpha=0$. Therefore, for a given $n$ and $k$, the value of $a_{n}$ will be a maximum when $\alpha=0$ i.e. the most slowly convergent series for $f(x)$ will arise in this case.

Let us estimate the value of $a_{n}$ for $\alpha=0$ by considering only the contribution from the two poles at $z= \pm i \beta$. From equation (10), with $M=2$, we find

$$
a_{n} \simeq \frac{1+(-1)^{n}}{i^{n} \pi \sqrt{\beta^{2}+1}\left(\beta+\sqrt{\beta^{2}+1}\right)^{n}} .
$$

When $n$ is odd, equation (12) gives immediately that $a_{n}=0$; when $n$ is even $(=2 r$, say $)$ we have,

$$
a_{2 r} \simeq \frac{2(-1)^{r}}{\pi \sqrt{\beta^{2}+1}\left(\beta+\sqrt{\beta^{2}+1}\right)^{2 r}} .
$$

The comparison of this estimate of $a_{2 r}$ with its actual value in the case when $k=1.2$ is given in Table 1 . For quite small values of $r$, it can be seen that these estimates are good.

Finally we can see qualitatively the effect of changing $k$. As $k$ becomes large, $\beta$ tends to zero and the poles approach the real axis. Thus $a_{n}$ for a given $n$ becomes larger as $k$ increases, and a higher degree polynomial is required to approximate $f(x)$ to the same accuracy.

4. A Pole of Order $k$. In Sections 2 and 3 we have considered only the case where $f(z)$ has simple poles at one or more points in the complex plane. Let us now consider the case where $f(z)$ has a pole of order $k$ at $z=z_{1}$ so that

$$
f(z)=\frac{A_{-k}}{\left(z-z_{1}\right)^{k}}
$$

where $A_{-k}$ is constant. If we write

$$
g(z)=\frac{1}{\sqrt{z^{2}-1}\left(z \pm \sqrt{z^{2}-1}\right)^{n}}
$$

then from equation (8) we find on using the same limiting process for the contour $C$, that

$$
a_{n}=-2 \times\left(\text { residue of } f(z) g(z) \text {, at } z=z_{1}\right),
$$

from which equations (13) and (14) give 


$$
a_{n}=-\frac{2 A_{-k}}{(k-1) !} g^{(k-1)}\left(z_{1}\right)
$$

The problem of determining $a_{n}$ is thus reduced to finding an explicit expression for the $(k-1)$ st derivative of $g(z)$. In order to do this we distinguish four cases, and will first consider the case when $\operatorname{Re} z_{1}>1$.

When $\operatorname{Re} z>1$, the correct choice of sign in $g(z)$ is the positive one in order that $\left|z \pm \sqrt{z^{2}-1}\right|$ should be $>1$. Now Erdélyi et al. [8, p. 240] gives

$$
g(z)=\frac{1}{\sqrt{z^{2}-1}\left(z+\sqrt{z^{2}-1}\right)^{n}}=\int_{0}^{\infty} e^{-z t} I_{n}(t) d t
$$

provided Re $z>1$, where $I_{n}(t)$ is the modified Bessel function of order $n$. Differentiating equation $(16),(k-1)$ times with respect to $z$, gives

$$
g^{(k-1)}(z)=(-1)^{k-1} \int_{0}^{\infty} t^{k-1} e^{-z t} I_{n}(t) d t .
$$

Again, from Erdélyi [8, p. 196], we find

$$
g^{(k-1)}(z)=\frac{(-1)^{k-1}(n+k-1) !}{\left(z^{2}-1\right)^{k / 2}} P_{k-1}^{-n}\left(\frac{z}{\sqrt{z^{2}-1}}\right)
$$

valid for $n \geqq 0$ and $k \geqq 1$, where $P_{k-1}^{-n}$ denotes the associated Legendre function of the first kind. Combining equations (15) and (17) we obtain the required result when $\operatorname{Re} z_{1}>1$,

$$
a_{n}=\frac{2(-1)^{k}(n+k-1) !}{(k-1) !\left(z_{1}^{2}-1\right)^{k / 2}} P_{k-1}^{-n}\left(\frac{z_{1}}{\sqrt{z_{1}^{2}-1}}\right) A_{-k} .
$$

An asymptotic form of $a_{n}$ can be given for large $n$. For example, Hobson [9, p. 308] gives

$$
P_{k-1}^{-n}\left(\frac{z_{1}}{\sqrt{z_{1}{ }^{2}-1}}\right)=\frac{1}{n !}\left(\frac{1}{z_{1}+\sqrt{\overline{z_{1}{ }^{2}-1}}}\right)^{n}\left[1+O\left(\frac{1}{n}\right)\right]
$$

which is valid provided

$$
\left|\frac{z_{1}}{\sqrt{z_{1}^{2}-1}}-1\right|<2
$$

Thus, subject to the condition on $z_{1}$ given by equation (20), we have for large $n$,

$$
a_{n} \simeq \frac{2(-1)^{k}(n+k-1) ! A_{-k}}{(k-1) !\left(z_{1}^{2}-1\right)^{k / 2} n !\left(z_{1}+\sqrt{\overline{z_{1}{ }^{2}-1}}\right)^{n}}, \quad\left(\operatorname{Re} z_{1}>1\right) .
$$

When $k=1$, this asymptotic form for $a_{n}$ is exact for all $n$. When $k=2$, we have that $a_{n}$ is given explicitly by

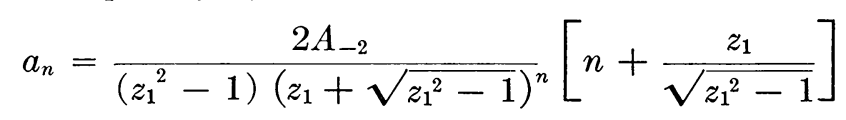

whereas equation (21) gives in this case

$$
a_{n} \simeq \frac{2 A_{-2}}{\left(z_{1}^{2}-1\right)\left(z_{1}+\sqrt{z_{1}^{2}-1}\right)^{n}}[n+1],
$$

which is quite a good approximation for values of $z_{1}$ not too close to $z=1$. 
We can proceed in a similar fashion when $\operatorname{Re} z_{1}<-1$, and we find

$$
a_{n}=\frac{2(-1)^{n+k}(n+k-1) ! A_{-k}}{(k-1) !\left(z_{1}^{2}-1\right)^{k / 2}} P_{k-1}^{-n}\left(\frac{z_{1}}{\sqrt{z_{1}^{2}-1}}\right),
$$

which agrees with a result previously given by Wimp [10] when $z_{1}$ is on the negative real axis such $z_{1}<-1$.

When $\left|\operatorname{Re} z_{1}\right| \leqq 1$, we again consider two cases depending upon whether $\operatorname{Im} z>0$ or $<0$. First let us consider the case when $\operatorname{Im} z>0$. Define $\zeta$ by

$$
z=\zeta e^{i \pi / 2}
$$

so that $\operatorname{Re} \zeta>0$. In terms of $\zeta, g(z)$ is given by

$$
g(z)=\frac{e^{-(n+1) i \pi / 2}}{\sqrt{\zeta^{2}+1}\left(\zeta+\sqrt{\zeta^{2}+1}\right)^{n}}
$$

the correct sign having been chosen, and from Erdélyi, et al. [2, p. 240] we have

$$
g(z)=e^{-(n+1) i \pi / 2} \int_{0}^{\infty} e^{-\zeta t} J_{n}(t) d t .
$$

This is valid for $n \geqq 0$, and $J_{n}(t)$ denotes the Bessel function of the first kind of order $n$. Differentiating equation $(24),(k-1)$ times with respect to $z$ and using the result given by Erdélyi et al. [2, p. 182] we find

$$
g^{(k-1)}(z)=\frac{(-1)^{k-1} e^{-(i \pi n / 2)}(k+n-1) !}{\left(z^{2}-1\right)^{k / 2}} P_{k-1}^{-n}\left(\frac{z}{\sqrt{z^{2}-1}}\right) .
$$

We can proceed similarly to find an analogous result for $g^{(k-1)}(z)$ when $\operatorname{Im} z<0$, by writing $z=\zeta e^{-(i \pi / 2)}$. From these results we find

$$
a_{n}=\frac{2(-1)^{k} e^{\mp(i \pi n / 2)}(k+n-1) ! A_{-k}}{(k-1) !\left(z_{1}^{2}-1\right)^{k / 2}} P_{k-1}^{-n}\left(\frac{z_{1}}{\sqrt{z_{1}^{2}-1}}\right)
$$

where the $\left(\begin{array}{c}\text { negative } \\ \text { positive }\end{array}\right)$ sign is chosen when $\operatorname{Im} z\left\{\begin{array}{l}>0 \\ <0\end{array}\right\}$ respectively.

Equations (18), (22) and (26) give the required values of the coefficient $a_{n}$ in the Chebyshev series expansion of the function $f(x)=\frac{A_{-k}}{\left(x-z_{1}\right)^{k}}$ for all possible values of $z_{1}$.

5. Entire Functions. So far we have considered only functions with poles in the complex plane. In this section we consider entire functions. We again take as our starting point equation (8), and choose as the contour $C$, the ellipse $E_{\rho}$. Consider the transformation

$$
z=\frac{1}{2}(\xi+1 / \xi)
$$

This maps the exterior of the ellipse $E_{\rho}$ in the $z$-plane onto the exterior of a circle $C_{\rho}$, of radius $\rho$ and centre the origin in the $\xi$-plane. With this transformation, we find,

$$
a_{n}=\frac{1}{\pi i} \int_{C_{\rho}} \frac{f\left[\frac{1}{2}\left(\xi+\frac{1}{\xi}\right)\right]}{\xi^{n+1}} d \xi
$$


If the Laurent expansion of $f\left[\frac{1}{2}\left(\xi+\frac{1}{\xi}\right)\right]$ is known, and in particular if the coefficient of $\xi^{n}$ is $\alpha_{n}$, then we have immediately $a_{n}=2 \alpha_{n}$. However, it is not likely in general that $\alpha_{n}$ will be known. We can then estimate an upper bound for $\left|a_{n}\right|$ quite simply. Suppose $M(\rho)$ denotes the maximum values of $|f|$ on the circle $C_{\rho}$ (or equivalently the ellipse $E_{\rho}$ ), then equation (28) gives immediately

$$
\left|a_{n}\right| \leqq \frac{2 M(\rho)}{\rho^{n}}
$$

By varying $\rho$, we find that value $\rho^{*}$ say, for which $2 M(\rho) / \rho^{n}$ is a minimum. We then have

$$
\left|a_{n}\right| \leqq \frac{2 M\left(\rho^{*}\right)}{\rho^{* n}}
$$

As an example, let us consider the function $e^{x}$. Now $e^{z}$ attains its maximum modulus on the ellipse $E_{\rho}$ at $z=a$, so we have $M(\rho)=e^{(1 / 2)\left(\rho+\rho^{-1}\right)}$. For a given $n$, the quantity $M(\rho) / \rho^{n}$ attains a minimum value at $\rho^{*}=n+\sqrt{n^{2}+1}$.

Thus

$$
\left|a_{n}\right| \leqq \frac{2 e^{\sqrt{n^{2}+1}}}{\left(n+\sqrt{n^{2}+1}\right)^{n}} \cong \frac{\sqrt{2 \pi n}}{2^{n-1} n !}
$$

for large values of $n$. It is of interest to compare this value with that obtained from equation (4), which in this case gives $\left|a_{n}\right| \leqq e / 2^{n-1} n$ !. This is a better approximation to $a_{n}$ than that given by equation (30). Since $e^{x}=2 \sum_{n=0}^{\infty \infty} I_{n}(1) T_{n}(x)$,

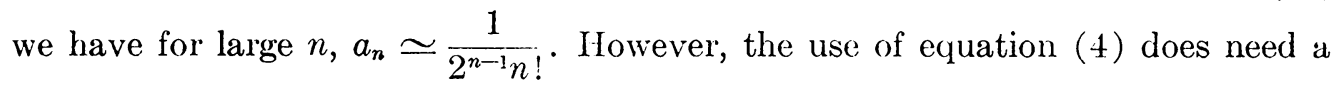
knowledge of the $n$th derivative of $f(x)$ and this is not in general as readily available as the value of $f(z)$ at points in the complex plane.

6. Functions Regular Except at $z= \pm 1$. We are not yet in a position to estimate the coefficients in the expansion of a function like $f(x)=\sqrt{1-x}$ which is regular everywhere except at the point $x=1$. In this section we shall derive such a result. The corresponding result for a function which is not regular at $x=-1$ will then be stated without proof.

Let us suppose that $f(x)=(1-x)^{\phi} g(x)$, where $\phi$ is not an integer and $g(x)$ is regular at $x=1$. Since $f(x)$ has, in particular, to be bounded at $x=1$, we have $\phi>0$. In equation (8), we shall now choose as the contour $C$, the ellipse $E_{0}$ described in the positive direction, a small circle $\gamma$ of radius $\epsilon$, centre +1 described negatively, and two line segments $A B$ and $C D$ where $A B$ is just below and parallel to the real axis, the point $A$ being on the ellipse $E_{\rho}$, the point $B$ on the circle $\gamma$; and $C D$ is just above and parallel to the real axis, the point $C$ being on the circle $\gamma, D$ on the ellipse $E_{\rho}$. The function $f(z)$ is regular within such a contour. We again assume that $f(z)$ is such that the integral over $E_{\rho}$ will tend to zero as $\rho$ becomes large. Since $\phi>0$, the integral around $\gamma$ will tend to zero as $\epsilon \rightarrow 0$. Thus in the limit as $\rho \rightarrow \infty$ and $\epsilon \rightarrow 0$, the only contributions to $a_{n}$ will come from the integrals along $A B$ and $C D$. Taking $f(z)=(1-z)^{\phi} g(z)$, we find that the two 
integrals along $A B$ and $C D$ combine to give

$$
a_{n}=-\frac{2 \sin \pi \phi}{\pi} \lim _{a \rightarrow \infty} \int_{1}^{a} \frac{|x-1|^{\phi} g(x) d x}{\sqrt{x^{2}-1}\left(x+\sqrt{x^{2}-1}\right)^{n}} .
$$

Putting $x=\cosh \theta$, if $a=\cosh \Theta$ then

$$
a_{n}=-\frac{2 \sin \pi \phi}{\pi} \lim _{\Theta \rightarrow \infty} \int_{0}^{\Theta}\left(2 \sinh ^{2} \frac{\theta}{2}\right)^{\phi} g(\cosh \theta) e^{-n \theta} d \theta .
$$

In order to approximate this integral, we assume that $n$ is chosen so large, and $g(x)$ is such that the main contribution to the integral comes from the values close to $\theta=0$. Replacing $\sinh \frac{\theta}{2}$ by $\frac{\theta}{2}$ and $g(x)$ by $g(1)$ we obtain

$$
a_{n} \simeq-2^{1-\phi} \frac{g(1) \cdot \sin (\pi \phi)}{\pi} \int_{0}^{\infty} \theta^{2 \phi} e^{-n \theta} d \theta
$$

Writing $t=n \theta$, we find

$$
a_{n} \simeq-\frac{2^{1-\phi} g(1) \cdot \sin (\pi \phi)}{\pi n^{2 \phi+1}} \Gamma(2 \phi+1)
$$

This is the required result for this case. As an example, consider again the function $f(x)=\sqrt{1-x}$. Now,

$$
\sqrt{1-x}=-\frac{4 \sqrt{2}}{\pi} \sum_{n=0}^{\prime} \frac{1}{4 n^{2}-1} T_{n}(x) .
$$

From equation (33) we have on putting $\phi=\frac{1}{2}$ and $g(1)=1$,

$$
a_{n} \simeq-\frac{\sqrt{2}}{\pi n^{2}}
$$

which compares very well with the exact result $a_{n}=-\sqrt{2} / \pi\left(n^{2}-\frac{1}{4}\right)$.

A similar result can be obtained for the point $z=-1$. If $f(x)=(1+x)^{\psi} h(x)$ where $h(x)$ is regular $x=-1$, then

$$
a_{n} \simeq-\frac{2^{1-\psi} \sin \pi \psi \cdot h(-1)}{\pi \cdot n^{2 \psi+1}}(-1)^{n} \Gamma(2 \psi+1) .
$$

These two results can be superposed if necessary, and as an example let us consider the estimate of $a_{n}$ for the function $f(x)=\sqrt{1-x^{2}}$. The function $f(z)=$ $\left(1-z^{2}\right)^{1 / 2}$ is regular everywhere except at $z= \pm 1$. Combining the results of equations (33) and (35) we find,

$$
a_{2 n} \simeq-\frac{1}{\pi n^{2}} \quad \text { and } \quad a_{2 n+1}=0
$$

which compares favorably with the actual values $a_{2 n}=-1 / \pi\left(n^{2}-\frac{1}{4}\right)$ and $a_{2 n+1}=0$.

7. Branch Point on Real Axis. The last case we shall consider is that where $f(z)$ has a branch point at $z=c$ on the real axis where $c>1$. As an example of such a function we have $f(x)=\sqrt{2-x}$, where there is a branch point of $f(z)$ at $z=2$. 
TABLE 2

\begin{tabular}{c|c|c}
\hline$n$ & estimated $a_{n}$ & actual $a_{n}$ \\
\hline 4 & -0.000497 & -0.000536 \\
5 & -0.000096 & -0.000100 \\
6 & -0.000019 & -0.000020 \\
7 & -0.000004 & -0.000004 \\
8 & -0.000001 & -0.000001 \\
\hline
\end{tabular}

Suppose that we have $f(z)=(c-z)^{\phi} g(z)$ where $\phi$ can be any number, not an integer, and $g(z)$ is regular at $z=c$. The contour $C$ is chosen in a way similar to that for the case when $f(z)$ has a branch point at $z=1$ but with the circle $\gamma$ enclosing the point $z=c$. In order that the integral around $\gamma$ should tend to zero when the radius tends to zero, we must have $\phi>-1$. Assuming also that the integral around the ellipse $E_{\rho}$ tends to zero as $\rho$ tends to infinity, we find on combining the contributions to the integral from the lines $A B$ and $C D$ that

$$
a_{n}=-\frac{2 \sin \pi \phi}{\pi} \lim _{a \rightarrow \infty} \int_{C}^{a} \frac{|x-c|^{\phi} g(x) d x}{\sqrt{x^{2}-1}\left(x+\sqrt{x^{2}-1}\right)^{n}} .
$$

As before, writing $x=\cosh \theta$, then if $c=\cosh \alpha$ we have

$$
a_{n}=-\frac{2 \sin \pi \phi}{\pi} \lim _{\Theta \rightarrow \infty} \int_{\alpha}^{\Theta}\left[2 \sinh \frac{\theta+\alpha}{2} \sinh \frac{\theta-\alpha}{2}\right]^{\phi} g(\cosh \theta) e^{-n \theta} d \theta .
$$

Again, considering that $n$ is chosen and $g(\cosh \theta)$ is such that the major contribution to the integral comes from around $\theta=\alpha$, we find, assuming that $(\theta-\alpha)$ is small,

$$
a_{n} \simeq-\frac{2 \sin \pi \phi(\sinh \alpha)^{\phi} g(c)}{\pi} \int_{\alpha}^{\infty}(\theta-\alpha)^{\phi} e^{-n \theta} d \theta
$$

On making the substitution $t=n(\theta-\alpha)$, we have the required result,

$$
a_{n} \simeq-\frac{2 \sin \pi \phi\left(c^{2}-1\right)^{\phi / 2} g(c) \Gamma(\phi+1)}{\pi n^{\phi+1}\left(c+\sqrt{c^{2}-1}\right)^{n}} .
$$

A comparison of this estimated value of $a_{n}$ with the actual value for the function $\sqrt{2-x}$ is given in Table 2 .

Once again, the estimated values obtained from equation (37) compare very well with the actual values.

Proceeding as above we can derive a similar result for the case when $f(z)$ has a branch point on the negative real axis at $z=-c,(c>1)$. If $f(z)=(c+z)^{\psi} h(z)$ where $h(z)$ is regular at $z=-c$, then for $\psi>-1$, we find for large $n$,

$$
a_{n} \simeq-\frac{2 \sin \pi \psi\left(c^{2}-1\right)^{\psi} h(-c)(-1)^{n} \Gamma(\psi+1)}{\pi n^{\psi+1}\left(c+\sqrt{c^{2}-1}\right)^{n}} .
$$

8. Extension to Expansions in Jacobi Polynomials. The foregoing analysis can be generalized to the case where $f(x)$, defined for $-1 \leqq x \leqq 1$, is expanded in a series of Jacobi polynomials $P_{n}^{(\alpha, \beta)}(x)$, see Szegö [11]. Suppose 


$$
f(x)=\sum_{n=0}^{\infty} a_{n} P_{n}^{(\alpha, \beta)}(x)
$$

then

$$
a_{n}=\frac{1}{h_{n}^{(\alpha, \beta)}} \int_{-1}^{1} w(x) P_{n}^{(\alpha, \beta)}(x) f^{\prime}(x) d x,
$$

where [11, equation (4.3.3)],

$$
h_{n}^{(\alpha, \beta)}=\frac{2^{(\alpha+\beta+1)} \Gamma(n+\alpha+1) \Gamma(n+\beta+1)}{(2 n+\alpha+\beta+1) \Gamma(n+1) \Gamma(n+\alpha+\beta+1)}
$$

and

$$
w(x)=(1-x)^{\alpha}(1+x)^{\beta} .
$$

Substituting for $f(x)$ from equation (5) into equation (39) we find

$$
a_{n}=\frac{1}{2 \pi i h_{n}^{(\alpha, \beta)}} \int_{C} f(z)\left\{\int_{-1}^{1} \frac{w(x) P_{n}^{(\alpha, \beta)}(x)}{z-x} d x\right\} d z .
$$

From [11, equation (4.61.4)] we have immediately

$$
a_{n}=\frac{1}{2 \pi i h_{n}{ }^{(\alpha, \beta)}} \int_{C} f(z) \cdot 2(z-1)^{\alpha}(z+1)^{\beta} Q_{n}{ }^{(\alpha, \beta)}(z) d z .
$$

Suppose now that $n$ is large; then a suitable asymptotic form for $Q_{n}{ }^{(\alpha, \beta)}(z)$ is given by (see Barrett, [12]),

$$
\begin{aligned}
2(z-1)^{\alpha}(z+1)^{\beta} Q_{n}{ }^{(\alpha, \beta)}(z) \simeq \sqrt{\frac{\pi}{n}} & \\
& \cdot \frac{2^{(\alpha+\beta+1) / 2}(z-1)^{(\alpha / 2)-(1 / 4)}(z+1)^{(\beta / 2)-(1 / 4)}}{\left(z \pm \sqrt{z^{2}-1}\right)^{n+\frac{1}{2}(\alpha+\beta+1)}},
\end{aligned}
$$

where the sign in $\left(z \pm \sqrt{z^{2}-1}\right)$ is chosen so that $\left|z \pm \sqrt{z^{2}-1}\right|>1$. This representation of $Q_{n}^{(\alpha, \beta)}(z)$ is valid in the plane cut along $[-1,1]$ with the neighborhood of the two points $z= \pm 1$ removed. Substituting equation (41) into (40) we have the required generalization of equation (8),

$$
a_{n} \simeq \frac{1}{2 \pi i h_{n}^{(\alpha, \beta)}} \sqrt{\frac{\pi}{n}} \cdot 2^{(\alpha+\beta+1) / 2} \int_{C} \frac{f(z)(z-1)^{(\alpha / 2)-(1 / 4)}(z+1)^{(\beta / 2)-(1 / 4)}}{\left(z \pm \sqrt{z^{2}-1}\right)^{n+\frac{1}{2}(\alpha+\beta+1)}} d z
$$

where $C$ is chosen so that $f(z)$ is regular within $C$. An analysis similar to that given in Sections 2-7, can be made on this equation.

9. Conclusion. In this paper we have considered estimates for the coefficients in the Chebyshev series expansion of a function $f(x)$. The form of the estimate depends upon whether $f(z)$ has poles, or has a branch point on the real axis including the end points $z= \pm 1$, or whether $f(x)$ is an entire function. The results may be superposed. Consider for example the function $f(x)=e^{x} /\left(1+x^{2}\right)$. This function has simple poles at $z= \pm i$, but the integral in equation (8) does not tend to zero around the ellipse $E_{\rho}$ as $\rho \rightarrow \infty$. Nevertheless an estimate to the coefficients $\left|a_{n}\right|$ can be found in this case by combining the results of Section 2 with the technique for 
entire functions as described in Section 5. Combining the results obtained in this way, estimates for the coefficients $a_{n}$ for large $n$ may be obtained with considerable accuracy for a large variety of functions.

Basser Computing Department,

School of Physics

University of Sydney

Sydney, N.S.W., Australia

1. C. W. Clenshaw \& A. R. Curtis, "A method for numerical integration on an automatic computer," Numer. Math., v. 2, 1960, p. 197.

2. C. W. Clenshaw, "The numerical solution of linear differential equations in Chebyshev series," Proc. Cambridge Philos. Soc., v. 53, 1957, p. 134.

3. L. Fox, "Chebyshev methods for ordinary differential equations," Computer J., v. 4, 1962, p. 318 .

4. C. W. Clenshaw \& H. J. Norton, "The solution of non-linear ordinary differential equations in Chebyshev series," Computer J., v. 6, 1963, p. 88.

5. D. ELLiotT, "The numerical solution of integral equations using Chebyshev polynomials," J. Math. Soc. Australia, v. 1, 1960, p. 344.

6. D. ElLIOTT, "A Chebyshev series method for the numerical solution of Fredholm integral equations," Computer J., v. 6, 1963, p. 102.

7. D. ElliotT, "A' method for the numerical integration of the one-dimensional heat equation using Chebyshev series," Proc. Cambridge Philos. Soc., v. 57, 1961, p. 823.

8. A. ERdélyi, W. Magnus, F. Oberhettinger \& F. G. Tricomi, Tables of Integral Transforms, v. 1. McGraw-Hill, New York, 1953.

9. E. W. HoBson, The Theory of Spherical and Ellipsoidal Harmonics, Cambridge Univ. Press, 1955 .

10. J. Wıмp, "Polynomial approximations to integral transforms," Math. Comp., v. 15, 1961, p. 174 1939

11. G. SzeGö, "Orthogonal polynomials," Amer. Math. Soc. Colloquium Publication v. 23,

12. W. BARRETT, "Convergence properties of Gaussian quadrature formulae," Computer J., v. 3, 1961, p. 272 . 